\title{
Isotope Ratios and Abundance Sensitivity Obtained with an Inductively Coupled Plasma-Time-of-Flight Mass Spectrometer
}

\author{
D. P. Myers, P. P. Mahoney, G. Li, and G. M. Hieftje \\ Department of Chemistry, Indiana Lniversity, Bloomington, Indiana. USA
}

\begin{abstract}
Isotope ratios and abundance sensitivities have been determined with an inductively coupled plasma-time-of-flight mass spectrometer (ICP-TOFMS). Abundance sensitivities are at least in the $10^{6}$ range for low abundance ions that precede high abundance ions. Three methods of detection for isotope-ratio measurement have been compared. The three systems involve gated detection followed by analog integration, analog averaging, or ion counting. Gated ion counting offers excellent precision - between 0.64 and $1.00 \%$ relative standard deviation (RSD). These values approach those predicted from counting statistics and are comparable to those reported for other inductively coupled plasma-mass spectrometry (ICP-MS) instruments. In addition, a greater number of accumulated counts or longer analysis times would afford precisions of $0.1 \%$ with stable gating electronics. The accuracy of the counting method is in the $1-10 \%$ range if no correction for mass bias is performed. However, this ion counting method suffers from a limited dynamic range due to pulse pileup. Constant-fraction discrimination gated integration and commercial boxcar averager techniques offer a broader dynamic range because of their analog nature, but the attainable RSD values are limited by drift in the detection systems and by the methods employed to calculate an accurate ratio. Overall, mass bias in the ICP-TOFMS is more severe than previous work in ICP-MS due primarily to detection system bias. (I Am Soc Mass Spectrom 1995, 6. 920-927)
\end{abstract}

I nductively coupled plasma mass spectrometry (ICP-MS) with quadrupole mass analyzers has been Lstudied and tested extensively for isotope-ratio analysis in a variety of fields that include geochemical and environmental applications $[1,2]$. However, recent workers have pointed out that its widespread use has been inhibited by its lack of precision $[0.1-1.0 \%$ relative standard deviation (RSD)] when compared to techniques such as thermal ionization mass spectrometry (TIMS), which offers precision levels better than $0.005 \%$ RSD [3]. The main disadvantage to TIMS is the long analysis times (up to 1 day) required to achieve the better precision [3]. Also, to obtain the highest precision $(0.1 \%)$ on ICP-MS quadrupole instruments requires rapid peak-hopping $[3,4]$, so the number of isotopes and elements that can be measured in a single run of fixed time is limited. Obviously, it would be advantageous to increase precision in routine ICP'-MS the sensitivity of the method trnables shorter analysis times and consequently higher sample throughput than alternative methods such as TIMS [5]. Typically the RSD values obtained in ICP-MS have been $2-3$ times higher than would be expected from counting statistic

Address reprint requests to Dr. Lar V. Heftie, Department at Chemistry, Indiana University, Blomingtom, IN $47+105$.
[3]. Instrument instability has been the major cause of the poorer precision $[3,6,7]$.

Recent work has focused on analysis of the sources of noise in ICP-MS and subsequent optimization of the instrument to reduce or eliminate the noise components $[3,4]$. For example, Furuta [4] has found that reduction of the dwell time between the measurement of individual isotope peaks can improve precision to values in the $0.1-0.3 \%$ RSD range by elimination of multiplicative noise components that exist in ICP-MS. At the shortest dwell times, on the order of $10 \mu \mathrm{s}$, precision was limited by Poisson statistics in the ion counts. Presumably, a larger number of sweeps could be accumulated to improve precision further. Begley and Sharp [3] used similar techniques to attain relative standard deviations of $0.05 \%$. Their studies showed also that better precision was possible by reduction of the measurement interval between isotopes. They suggest that a multicollector mass spectrometer should exhibit even better precision than that seen with the sequential quadrupole mass analyzer, as long as the noise components in each signal remain multiplicative. In accordance with this explanation, ratios have been measured with a double-focusing magnetic sector mass analyzer equipped with seven Faraday collectors for simultaneous detection of masses [8]. This mass analyzer also benefits from flat-topped symmetrical peaks, 
which allow more accurate determination of peak heights than is convenient with the rounded peaks from quadrupole filters [8]. Isotope ratios obtained with this sector instrument have RSDs in the range of $0.014-0.022 \%$ and an error (accuracy) of $0.01 \%$ [8-10]. Of course, the limitation of such a multidetector simultaneous instrument is the number of isotopes and elements that can be analyzed in an available measure. ment period. Small or transient samples could not be analyzed for more than a few isotope ratios for a very few elements. Clearly, a fast mass analyzer that samples or measures all of the atomic ions simultaneously seems better suited to general-purpose isotope-ratio analyses.

A time-of-flight mass spectrometer does not measure ion signals at exactly the same time. However, all ions are sampled simultaneously from the inductively coupled plasma (ICP) in each cycle of mass analysis. Therefore, provided that the sampling process is reproducible, drift and multiplicative noise in the source can be removed from the individual ion signals. Signals also are measured at the same detector, so its noise should be nearly identical for all ratioed signals. In fact, isotope-ratio measurements with time-of-flight mass spectrometers already have shown promise with ionization techniques such as resonance ionization [11-13], laser ablation [14], and secondary ion mass spectrometry [15]. Measurement precision in these studies was in the range of 0.3 to $1.0 \%$ relative standard deviation, limited mainly by counting statistics at the best precision levels [11]. Because the peak shape in time-of-flight mass spectrometry (TOFMS) is also rounded, just as with a quadrupole filter, a detection system that integrates the ion peaks would be advantageous, as already has been demonstrated with a gated pulse counter [11].

\section{Experimental}

The ICP-TOFMS instrumentation is identical to that described previously [16-18]. For all data presented here the ICP-TOFMS was operated in the reflectron mode because of the higher resolving power it offered. In addition, the electrostatic quadrupole lens was operated in the pulsed ion-injection mode [17], at an instrumental repetition rate of $7.1 \mathrm{kHz}$. All other instrument conditions are identical to those described previously [18]. In this investigation we utilize and compare three systems for isotope-ratio measurement: (1) commercial boxcar averagers, (2) constant-fraction discriminator (CFD)-gated integrator [19], and (3) gated ion counting. All of these detection methods were described in detail previously [18]. A Tektronix (Beaverton, OR) TDS 520 digital oscilloscope, which is normally used to acquire full time-of-flight mass spectra, was ineffective to attain acceptable isotope-ratio precision.

For the foregoing methods, the two isotopes of interest were isolated by two separate timing gates of 30-50-ns duration each; optimization of gate width and delay time is critical to achieve optimal performance of all systems. In the case of the boxcar averagers and CFD-gated integrators, the analog outputs of the two measurement channels are ratioed. In the CFD-gated integrator the ratio is performed by using a Vational Instruments NB-MIO-16XL analog-to-digital converter board and LabVIEW II software (National lnstruments, Austin, TX). With the boxcar averagers the output of both averagers is sent to the ratio function of a Stanford Research Systems Model SR235 ana$\log$ processor; the output of this module is read out on the National Instruments board

When the boxcar averagers were used, both modules (Stanford Research Model SR250) were set to the same sensitivity; however, to account for gain difference between the averagers, the gates were switched in their mass (time) after an initial measurement and the ratio was determined again. The two measured ratios were inserted into the following equation to obtain the gain-corrected value:

$$
\text { actual ratio }=(\text { ratio } 1 \times \text { ratio } 2)^{1 / 2}
$$

The same method and calculation were employed with the CFD-gated integrator system. Anticipated uncertainties in these measurements were computed based on error propagation of the precision of the individual values.

The gated counter method is similar to a setup described by Green et. al. [11]. Two timing gates (optimized at widths between 30 and $50 \mathrm{~ns}$ ) are used to trigger constant-fraction discriminators (CFDs: Oxford Instruments, Oak Ridge, TN) that count ion hits above a certain threshold and output nuclear instrument module (NIM) pulses. The output of the CFD is connected to a Hewlett-Packard (Avondale, PA; model $5302 ; 50-\mathrm{MHz}$ ) universal counter. The ratio measurements are performed by the counter, which reads out the counts of one channel that occur for a selected number of counts $\left(1 \times 10^{1}-1 \times 10^{6}\right)$ in the other. To prevent pulse pileup with this method, the rate of ion counts in each channel should be maintained at less than 0.1 ions per repeller pulse. However, higher concentrations that produce between 0.1 and 5 ions per repeller pulse were used in some cases to reduce the analysis times. Accurate corrections for pulse pileup are possible when standard samples of known isotopic composition are used in the same concentration range as the unknown samples.

In the ratio measurements here no corrections were made for mass bias in the instrument; however, in all of the methods such corrections are possible. All ratio determinations were made on lead isotopic SRM 981 (National Institute of Standards and Technology, Gaithersburg, MD), which was dissolved in nitric acid to produce a stock solution (1309 Ppm) for subsequent dilution. The certified values for this lead standard are listed in Table 1. The concentrations ultimately used for isotope-ratio analyses were $1.31 \mathrm{ppm}$ and 262, 196 , and $62 \mathrm{ppb}$. 
Table 1. Certified isotope ratios for SRM-981"

\begin{tabular}{|c|c|}
\hline${ }^{204} \mathrm{~Pb} /{ }^{206} \mathrm{~Pb}$ & $0.059042 \pm 0.000037$ \\
\hline${ }^{207} \mathrm{~Pb} /{ }^{206} \mathrm{~Pb}$ & $0.91464 \pm 0.00033$ \\
\hline${ }^{208} \mathrm{~Pb} /{ }^{206} \mathrm{~Pb}$ & $2.1681 \pm 0.0008$ \\
\hline
\end{tabular}

${ }^{a}$ Source: National Institute of Standards and Technology

To measure the abundance sensitivity in the ICPTOFMS, an averaged spectrum, accumulated from 10,000individual scans, was obtained for both a solution of 1000-ppm Bi (Johnson Matthey, Ward Hill, MA) and a blank solution. These spectra were recorded on the TDS 520 digital oscilloscope.

\section{Results and Discussion}

\section{Abundance Sensitivity}

Values for resolving power measured with this ICPTOFMS have ranged from 1400-2300 [full width at half-maximum (fwhm)] [17], which would suggest high levels of abundance sensitivity. However, abundance sensitivity depends not only on resolving power but also on mass-spectral peak shape. Figure la shows a spectrum for a solution that contains an equal concentration of $\mathrm{Pb}$ and $\mathrm{Bi}(4 \mathrm{ppm})$ that exhibits a resolving power of $\sim 1700(\mathrm{fwhm})$. The peaks are generally well shaped, but with obvious ringing on the high mass side, and are baseline-resolved on the mass axis. In the flight-time domain the individual ion peaks range from 20 to $40 \mathrm{~ns}$ at the base, which allows gated detection of single masses.

The abundance sensitivity of a mass analyzer is an important indicator of the ability to determine low abundance isotopes that are adjacent to very abundant isotopes. Quadrupole mass analyzers possess abundance sensitivities of $10^{5}-10^{6}$. For the ICP-TOFMS, Figure $1 \mathrm{a}$ and $\mathrm{b}$ shows a spectrum obtained with the ICP-TOFMS over the mass range 204-212 for a solution of 1000 -ppm Bi. Both spectra were acquired without preamplification because the large $\mathrm{Bi}$ signal would saturate the amplifier output. A spectrum of the blank solution $\left(5 \% \mathrm{HNO}_{3}\right)$ also was obtained but is not displayed in Figure 1. At the adjacent mass M-1 (208 u) no measurable difference was observed between the $\mathrm{Bi}$ spectrum and the blank. However, the average background level of $0.35901 \mathrm{~V}$ and its standard deviation of $0.0013598 \mathrm{~V}$ can be used to estimate the minimum detectable concentration that would yield a signal of $3 \sigma$ or $0.0041 \mathrm{~V}$. The sensitivity from Figure 1a for ${ }^{208} \mathrm{~Pb}$ is $30 \mathrm{mV} / \mathrm{ppm}$; thus, this detectable level corresponds to $\mathrm{a} \mathrm{Pb}$ concentration of approximately 140 ppb. Use of the signal level for 1000-ppm Bi minus the blank level and division by the minimum detectable level of $0.0041 \mathrm{~V}$, yields a ratio of 562.7 . When the concentration difference between the $\mathrm{Bi}$ and $\mathrm{Pb}$ is taken into account, an abundance sensitivity of $4 \times 10^{6}$ is calculated. Based on the preceding treatment we can
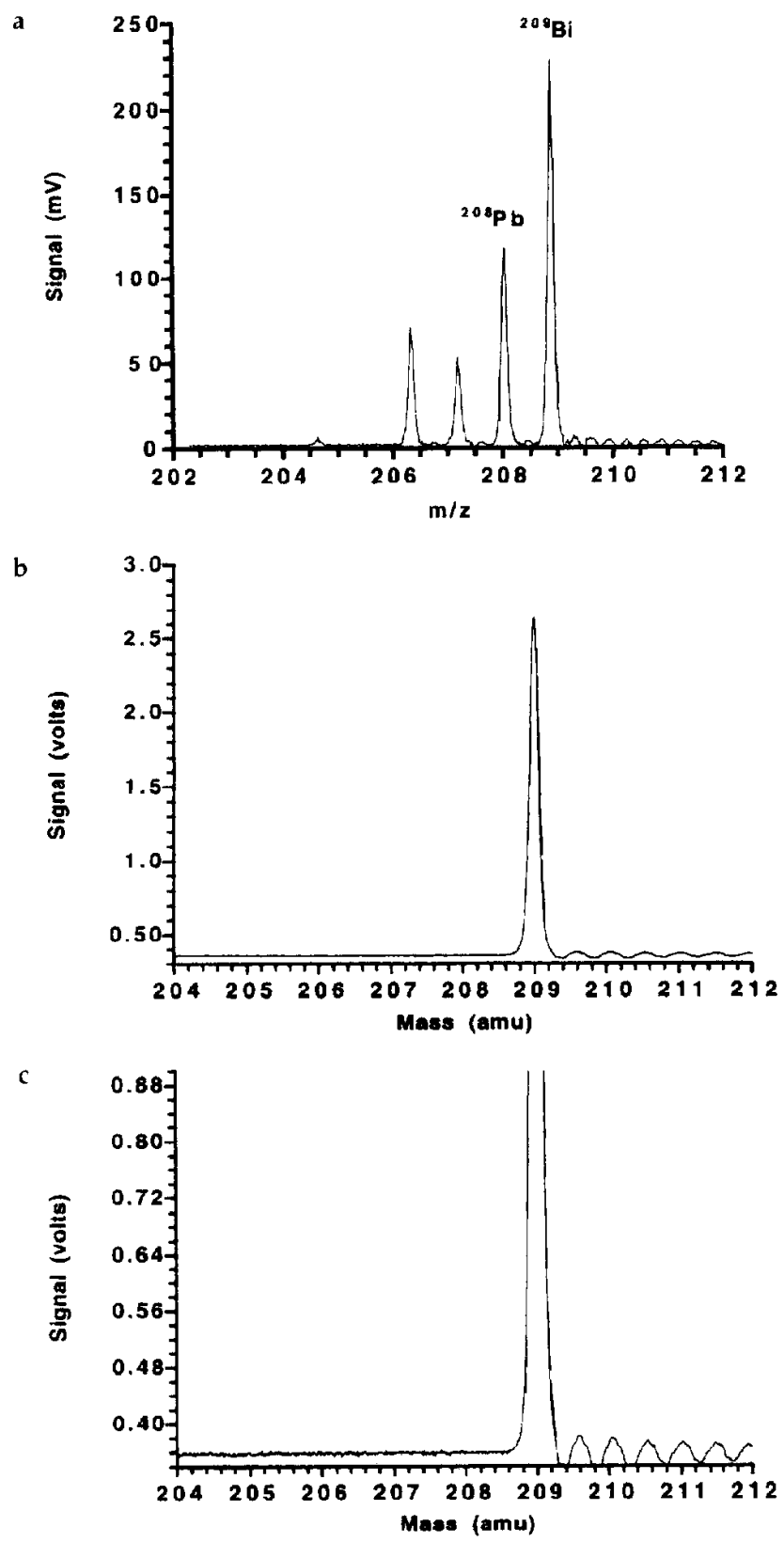

Figure 1. (a) ICP-TOFMS spectrum for a 4-ppm solution of both $\mathrm{Pb}$ and $\mathrm{Bi}$ by using the ion reflectron (average of 1000 shots). (b) Spectrum of 1000 -ppm $\mathrm{Bi}$ (average of 10,000 shots) with no preamplification. (c) Expanded scale of spectrum in (b).

state only that the lower limit of abundance sensitivity is at this value $\left(4 \times 10^{6}\right)$, because the contributed amount of $\mathrm{Bi}$ at adjacent mass 208 is not detectable in our spectrum.

The expanded scale in Figure $1 \mathrm{~b}$ reveals a problem in ICP-TOFMS: very intense ion signals can cause saturation of the MCP and ringing at the anode output that would preclude the analysis of a less abundant ion 1 mass unit heavier than the more abundant mass. Also, there is a difference in baseline before and after the $\mathrm{Bi}$ peak due to saturation of the microchannel plate (MCP) detector. A similar problem was noted in a 
laser microprobe mass analyzer, but was caused by asymmetry in the peaks and was not as severe [12]. The incorporation of a different detector and improved impedance-matching system might alleviate the ringing; however, more than likely, a detector such as a discrete dynode electron multiplier might be necessary to overcome the saturation problem.

\section{Isotope-Ratio Analysis}

\section{Gated Ion Counting}

With the gated counting method, isotope ratios from the SRM-981 standard were determined for a range of accumulated counts of the less abundant isotope. Table 2 lists the measured values for three $\mathrm{Pb}$ isotope ratios. As expected, at a greater total number of counts the precision of the measurement improved due to counting statistics. This trend is evident in Table 2, where the RSD for the measured ratios clearly improves in all cases as the number of total counts increases. Extrapolation to $1,000,000$ counts and use of the experimentally verified dependence of RSD on $1 /$ (number of counts $)^{1 / 2}$ enabled the precision to be computed as $0.12 \%$, approximately what would be expected from counting statistics $(0.1 \%)$. Of course, to acquire this number of counts would require $3-8 \mathrm{~h}$ at the signal levels employed in this experiment (recall that signal levels are necessarily low to avoid pulse pileup).

A disadvantage to counting measurements is the long analysis time required to produce better preci- sion. Moreover, for such extended analysis times, gate jitter and drift are likely to degrade the precision eventually. A system with stable gates would alleviate this problem; however, truly excellent precision $(<0.01 \%)$ would still require a long measurement time. One way to shorten the analysis time would be to increase the repetition rate of the TOFMS, which could approach $20 \mathrm{kHz}$ with the current arrangement $[16,18]$. 'This modification would reduce analysis times by a factor of approximately 3 .

For both the 208/206 and 207/206 ratio measurements the concentration used was $62 \mathrm{ppb}$ except in the case where 100,000 counts of the less abundant isotope were collected; in that situation a 196-ppb solution was employed. The analysis time for the 196-ppb solution was approximately $2-5 \mathrm{~min}$ for each determination. Even though the ratio at this number of accumulated counts is more precise than when 10,000 counts were accumulated, accuracy is sacrificed somewhat because ${ }^{208} \mathrm{~Pb}$ signals pile up $(>2$ ions per repeller pulse count rate), whereas ${ }^{206} \mathrm{~Pb}$ counts do not. As a consequence, the measured ratio of $2.0347 \pm 0.0130$ is lower than the certified value of $2.1681 \pm 0.0008$. The $207 / 206$ ratio experiences the same problem because both isotopes approach pileup conditions $(\sim 0.1$ ion per repeller pulse). However, for values determined at 10,000 counts, both ratios agree with the certified values within experimental precision, as does the value for the 204/206 ratio. A more detailed discussion of precision and mass bias will be offered in a later section.

The limit of precision for the isotope ratio $\mathrm{A} / \mathrm{B}$ from counting statistics can be determined from the follow-

Table 2. Isotope ratio results for the gated ion counting system ${ }^{\mathrm{a}}$

\begin{tabular}{|c|c|c|c|c|}
\hline $\begin{array}{l}\text { Ratio (A/B) } \\
\text { concentration }\end{array}$ & Counts B & Average $(n=24)$ & $\begin{array}{l}\text { RSD } \\
(\%)\end{array}$ & $\begin{array}{c}\text { RSD } \\
\text { Limit }^{\text {y }} \\
(\%)\end{array}$ \\
\hline \multicolumn{5}{|l|}{$\overline{204} \mathrm{~Pb} /{ }^{206} \mathrm{~Pb}$} \\
\hline \multirow[t]{3}{*}{ 196-pph SRM-981 } & 100 & $0.0672 \pm 0.0051$ & 7.6 & 10.3 \\
\hline & 1,000 & $0.0663 \pm 0.0021$ & 3.2 & 3.3 \\
\hline & 10,000 & $0.0650 \pm 0.0011$ & 1.7 & 1.0 \\
\hline \multicolumn{5}{|l|}{${ }^{207} \mathrm{~Pb} /{ }^{206} \mathrm{~Pb}$} \\
\hline \multirow[t]{3}{*}{ 62-ppb SRM-981 } & 100 & $0.8921 \pm 0.1076$ & 12.1 & 14.6 \\
\hline & 1,000 & $0.9067 \pm 0.0392$ & 4.3 & 4.6 \\
\hline & 10,000 & $0.9290 \pm 0.0217$ & 2.3 & 1.4 \\
\hline 196-ppb SRM-981 & 100,000 & $0.8998 \pm 0.0095$ & 1.0 & 0.46 \\
\hline \multicolumn{5}{|l|}{${ }^{208} \mathrm{~Pb} /{ }^{206} \mathrm{~Pb}$} \\
\hline \multirow[t]{3}{*}{ 62-ppb SRM-981 } & 100 & $1.8251 \pm 0.4182$ & 22.9 & 16.5 \\
\hline & 1,000 & $2.1145 \pm 0.1221$ & 5.8 & 5.5 \\
\hline & 10,000 & $2.0886 \pm 0.0387$ & 1.8 & 1.8 \\
\hline 196-ppb SRM-981 & 100,000 & $2.0347 \pm 0.0130$ & 0.64 & 0.55 \\
\hline
\end{tabular}


ing equation:

relative standard deviation

$$
=\left(\mathrm{A}^{1 / 2} / \mathrm{A}+\mathrm{B}^{1 / 2} / \mathrm{B}\right)^{1 / 2} \times 100
$$

where $A$ is the average number of counts in the more abundant channel and $B$ is the number of counts in the less abundant channel (10-100,000 in these cases). Table 2 shows the limit of precision attainable in each measurement based on the number of counts accumulated for the less abundant isotope. Most measured values approach precision limits dictated by counting statistics and in some cases are better than those predicted, due to the uncertainty in the small number of measured values $(n=24)$. In most cases, the larger number of accumulated counts exhibits precision lev. els poorer than the counting-statistics limit. Long term temporal drift in the gates contributes to this poorer precision, but there might also be cross talk between the two gating channels. Note in Table 2 that the measured RSD for the 207/206 ratio is two times higher than the RSD limit, whereas the 208/206 ratio is closer to the limit, even though they both require similar analysis times. This observation may suggest that there is more jitter or drift from interchannel cross talk when the gate signals are close together in time.

Overall, the precision values are as close to counting-statistics limits as those obtained with quadrupole mass analyzers $[3,4]$. In general, the precision offered by the gated ion-counting method is excellent, especially when 100,000 or more counts of the less abundant isotope are accumulated. For analysis times restricted to less than $5 \mathrm{~min}$ for a single measurement, pulse pileup must be endured in the 208/206 and $207 / 206$ ratios if the best precision is to be obtained.
Because of pulse pileup, gated ion counting would probably be attractive over a range of only 2-3 orders of magnitude in concentration with the current system.

\section{Boxcar Averagers}

Boxcar averagers provide the ability to average up to 10,000 shots of the TOFMS signal in a single channel. In addition, such analog measurements broaden the dynamic range and remove the problem of pulse pileup, both of which hinder the counting technique described earlier. With the averaging capability the precision of the ratio can be improved considerably. Table 3 lists the results obtained with the averagers for lead 208/206 and 207/206 ratios. The sensitivity settings of both channels were identical for the ratios (207/206 and 208/206) measured here so that the precision levels can be compared with confidence.

As discussed in the Experimental section the gaincorrected ratio is determined by switching the gates of the averagers and use of $\mathrm{eq} 1$ to calculate the true ratio from two individual ratio measurements. The averages of 100 measurements (each taking approximately 1.4 s) of each ratio (first directly and then with switched channels) are listed in columns labeled Ratio 1 and Ratio 2 of Table 3. The ratios calculated from eq 1 for eight determinations in the 208/206 case and five determinations for $207 / 206$ are listed in the third column. These calculated ratios have associated with them the propagated uncertainties from the individual ratio measurements (columns Ratio 1 and Ratio 2). The averages of all the calculated ratios of both 208/206 and $207 / 206$ are shown at the bottom of column 3 in Table 3.

Table 3. Isotope ratio results for the boxcar averagers"

\begin{tabular}{lcccc}
\hline & Ratio 1 & Ratio 2 & Calculated & $\begin{array}{c}\text { RSD } \\
\text { (\%) }\end{array}$ \\
\hline${ }^{208 \mathrm{~Pb} /{ }^{206} \mathrm{~Pb}}$ & $1.681 \pm 0.020$ & $2.631 \pm 0.042$ & $2.103 \pm 0.019$ & 0.90 \\
& $1.689 \pm 0.027$ & $2.634 \pm 0.055$ & $2.109 \pm 0.026$ & 1.23 \\
& $1.751+0.036$ & $2.533 \pm 0.059$ & $2.106 \pm 0.030$ & 1.42 \\
& $1.750 \pm 0.019$ & $2.807 \pm 0.047$ & $2.216 \pm 0.021$ & 0.95 \\
& $1.671 \pm 0.035$ & $2.706 \pm 0.038$ & $2.126 \pm 0.023$ & 1.08 \\
& $1.651 \pm 0.059$ & $2.684 \pm 0.058$ & $2.105 \pm 0.037$ & 1.75 \\
& $1.782 \pm 0.021$ & $2.660 \pm 0.062$ & $2.177 \pm 0.027$ & 1.24 \\
& $1.788 \pm 0.016$ & $2.350 \pm 0.076$ & $2.050 \pm 0.034$ & 1.66 \\
Average & & & $2.124 \pm 0.062$ & 2.92 \\
${ }^{207} \mathrm{~Pb} /{ }^{206} \mathrm{~Pb}$ & $1.289 \pm 0.025$ & $0.778 \pm 0.015$ & $1.001 \pm 0.014$ & 1.37 \\
& $1.402 \pm 0.034$ & $0.784 \pm 0.017$ & $1.048 \pm 0.011$ & 1.05 \\
& $1.389 \pm 0.046$ & $0.572 \pm 0.012$ & $0.891 \pm 0.010$ & 1.12 \\
& $1.235 \pm 0.021$ & $0.588 \pm 0.011$ & $0.852 \pm 0.008$ & 0.94 \\
& $1.224 \pm 0.024$ & $0.842 \pm 0.019$ & $1.015 \pm 0.011$ & 1.08 \\
Average & & & $0.961 \pm 0.085$ & 8.82 \\
\hline
\end{tabular}

\footnotetext{
a $1.31-$ ppm SRM $-981: 50$-ns gate; 10,000 averages: $n=100$ for each ratio
} 
For the 208/206 ratio the individual calculated values exhibit precision in the range of 0.9 to $2.00 \% \mathrm{RSD}$, which is about 10 times worse than the best isotoperatio precision reported for peak-hopping quadrupole mass spectrometers $[3,4]$. However, our measurements (each 10,000 averages) were obtained over integration times of only $1.4 \mathrm{~s}$, whereas the previous studies [3, 7] used times close to 1 min. Moreover, the system used here should be able to produce any number of isotope ratios simultaneously for a multielement solution. Because of this study's shorter measurement times, the RSD values reported here would be expected to be higher than were reported from earlier studies with simultaneous instruments such as the double-focusing multiple collector ICP-MS [8-10], with which measurement times were 5-10 $\$[9,10]$. The run-to-run variation in the present instrument is reflected in the average $208 / 206$ precision value cited in Table $3(2.92 \%)$. This longer term instability probably results in part from switching of the boxcar channels to compensate for their gain difference. When the channels are switched, there is a brief change in the dc offset of the averagers. Evidence for this occurrence can be seen in Figure 2, which shows the time-dependent variation in the two measured ratios immediately after the channels are switched. Clearly, the first few values at the beginning of each measurement are affected by this brief offset and serve to lower the precision considerably. In the future, a better way to compensate for interchannel gain will have to be found.

In the case of the lead $207 / 206$ ratio the precision is approximately of the same magnitude as that for the $208 / 206$ determination, but the variation in all calculated ratios is higher at $8.8 \%$, which indicates that the run-to-run precision is worse for this ratio. Reasons for this disparity are unknown at this time. Both averages of all calculated values agree with the certified values (see Table 1) within their precision. The individual

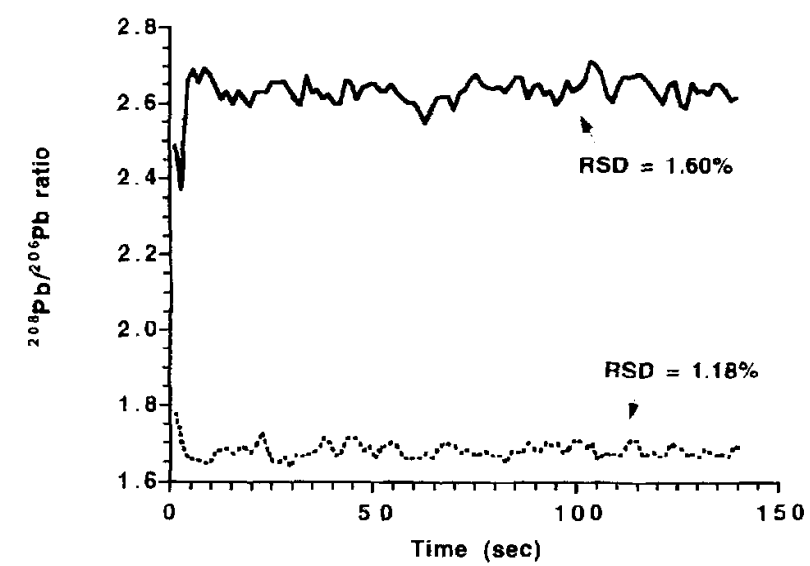

Figure 2. Effect of chamel switching on the precision of the isotope-ratio measurements. Each trace show's the time-dependent averaged ratios $(10,000$ averages for each plotted point produced by the boxcar averager immediately after the channels were switched. The top trace was obtained first, the channels were switched, and then the bottom trace was rourded. precision values, especially in the $208 / 206$ case, are quite promising but are probably limited by independent drift in the two analog channels used to obtain the ratio. In addition, the switch of channels during ratio analysis certainly degrades the run-to-run precision.

Individual listed ratio values (first two columns in Table 3) represent the means of 100 measurements, each of which is obtained from 10,000 boxcar averages. As would be expected, the precision of these determinations increases with the number of averages. We observed earlier [18] that the RSD of the ${ }^{208} \mathrm{~Pb} /{ }^{206} \mathrm{~Pb}$ ratio improves roughly as the square root of the number of boxcar averages. With the number of boxcar averages set to 10,000 (the limit of the SRS250 instrument), the precision can be as good as $0.57 \%$ [18]. If the average of 10 of these 10,000-pulse averages $(100,000$ averages $)$ is taken, the RSD improves to $0.47 \%$ [18]. The 100,000-averages value asymptotically seems to approach a limit in precision for the boxcar-averager system. The limit at this point is dictated mainly by the stability of the boxcar-averager output $(0.01 \% / \mathrm{min})$ and by independent drift in the two boxcar channels.

In this earlier study, there was no attempt to correct for mass bias in the two electronic measurement channels, so there was no need to switch them. The only adjustment was to optimize the boxcars and zero them. A comparison of these results with those of the present study suggests that the channel-switching method adopted here should be avoided.

In summary, the boxcar averagers offer an efficient and fast method of isotope-ratio analysis. In these investigations, each individual isotope ratio took about 5-6 min to ascertain, a time limited mainly by the channel switching used to compensate for differences in averager response. The precision is fair, provided drift from run to run can be eliminated. A possible way to remove this drift would be to calibrate the ratio from the two channels based on a standard such as SRM-981, then to use these two channels exclusively for the same isotopes. In this way, channel switching could be avoided.

The possibility exists with boxcar averagers (or any gated analog detection system) to monitor many ratios simultaneously simply by using a greater number of channels. Such a setup also would permit normalization to other isotope ratios, possibly to improve precision further, as has been demonstrated by others who used a simultaneous multichannel mass spectrometer [8]. A multichannel gated analog system would take best advantage of the speed of the TOFMS and should offer precision at least as good as that for isotope-ratio measurements made on typical ICP-MS quadrupole systems.

\section{CFD-Gated Integrators}

The CFD-gated integrator system has produced promising detection limits through signal-to-noise ra- 
tio enhancement $[18,19]$. As a result, the precision of this system was compared to that of the boxcar averagers and gated ion counting. However, we have observed that the precision of the CFD-gated integrators is limited to about 1-2\% RSD. Evidence for this limit is shown in Figure 3, where the RSD for the ${ }^{208} \mathrm{~Pb} /{ }^{21 \%} \mathrm{~Pb}$ ratio is plotted as a function of integration time for a single ratio measurement (i.e., no switching of integrator channels). The precision of the ratio does not improve appreciably beyond an integration time of $20 \mathrm{~s}$, at which point it is approximately $1 \%$ RSD.

This limited precision can be traced to the stability of the two integrator channels. Figure 4a shows the signal stability over a 5 -min period produced by the CFD-gated integrator (20-s integration) for a stable dc signal $(-70 \mathrm{mV})$ and at several gating frequencies. Because the integrator gate is ordinarily opened by a discriminator pulse only when a signal is present [19], the gating operation necessarily bypassed the discriminator. Figure $4 \mathrm{~b}$ shows the signals from the two channels and their ratio at the $2.1-\mathrm{kHz}$ gating frequency (which exhibited the poorest precision in Figure 4a). Overall, the precision of each integrator is in the $0.4-0.8 \%$ RSD range and for the ratio of the two channels (Figure $4 \mathrm{~b}$ ) the RSD is $0.72 \%$ in the $2.1-\mathrm{kHz}$ case. The precision has a similar magnitude at all other triggering frequencies.

If we assume the drift in the two channels is independent, the precision for the ratio in Figure $4 b$ should be $\left.1.0 \%\left[\left(0.9^{2}+0.5^{2}\right)^{1 / 2}\right)\right]$; however, as can be seen in Figure $4 b$, the drift in the two channels is somewhat correlated, so the precision is better than this prediction. Regardless, based on the precision in Figure 4 for stable dc signals, it is unlikely that the gated integrator will yield RSD values better than $1 \%$ in TOFMS. Because this level of precision is considerably lower than was achieved with the commercial boxcar averagers, there must be additional sources of noise and drift in

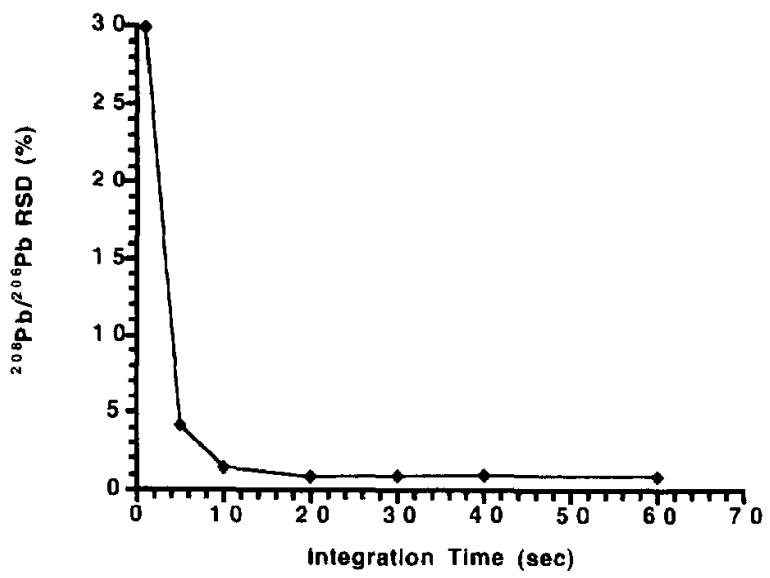

Figure 3. Fffect of integration time on relative standard devia tion (RSD) of a single ${ }^{2018} \mathrm{~Pb},{ }^{206} \mathrm{~Pb}$ ratio (SRM-981 standard) measurement (columns Ratio 1 and Ratio 2 in Table 3) for the CFD-gated integrator system. No channel switching was employed.
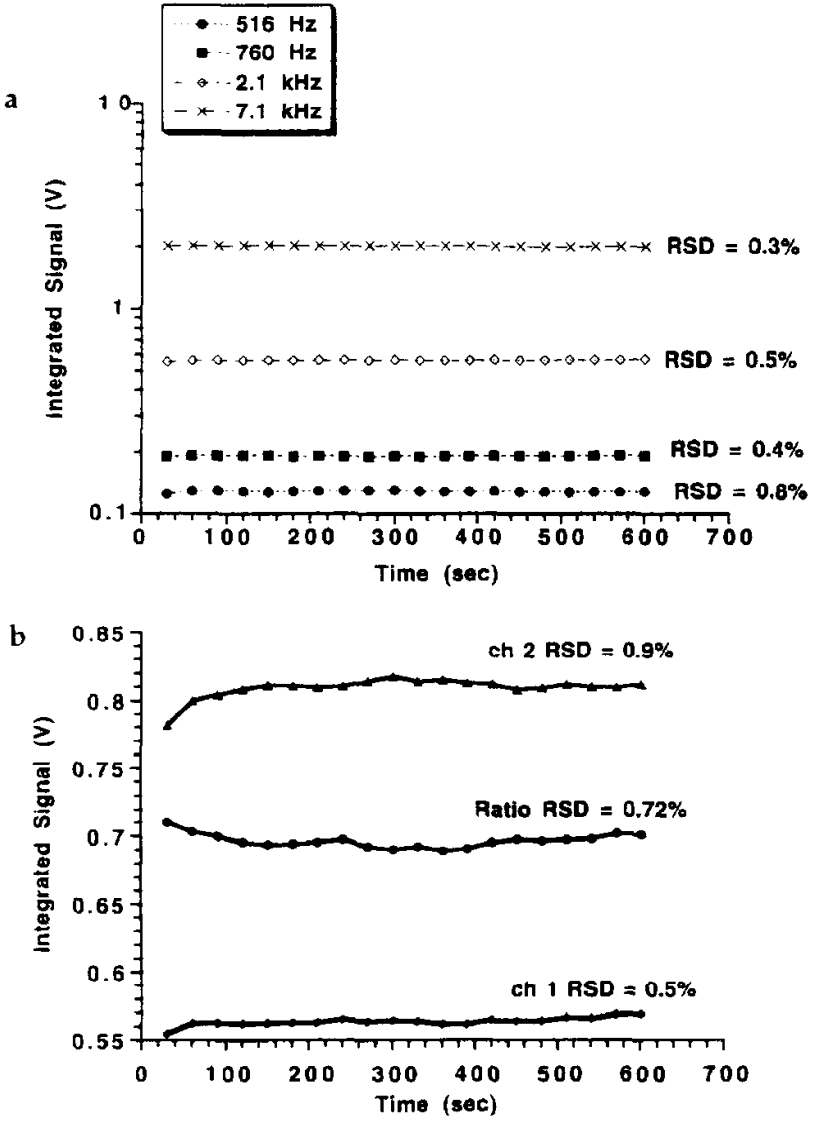

Figure 4. Integrated signal output (20-s integration time) from the CFD-gated integrators for a dc signal of $-70 \mathrm{mV}$ as a function of time. (a) Integrated signals for varied gating frequencies. (b) Integrated signals from both channels and their ratio for the 2.1-kHz gating frequency.

the CFD-gated integrator. One likely source is timing jitter. 'I he timing gates used in the gated integrator are hardly state-of-the-art, whereas those in the averagers are stable to tenths of nanoseconds.

\section{Mass Bias in the Lead Isotope Ratios}

Mass discrimination in the ratio measurements can be estimated and, if necessary, corrected with the following equation [9]:

$$
R_{\text {true }}=R_{\text {meas }}(1+C)^{\delta m}
$$

in which $R_{\text {true }}$ is the true ratio value, $R_{\text {meas }}$ is the measured ratio value, $C$ is the bias factor, and $\delta m$ is the mass difference. Mass bias was evaluated in the ICP-TOFMS for each detection system described previously. Table 4 lists the calculated $C$ values based on the values in Tables 2 and 3 . Clearly, mass bias is strongly dependent on the chosen detection system. The worst mass bias occurs in the CFD-gated integrator, which is not surprising because of its poor stability. Gated ion counting and the commercial boxcar averager offer the lowest levels of mass bias. 
Table 4. Mass bias factors in the JCP-TOFMS for the measurement systems employed

\begin{tabular}{lr}
\hline & \multicolumn{1}{c}{$C$} \\
\hline \hline Gated ion counting \\
${ }^{204} \mathrm{~Pb} /{ }^{206} \mathrm{~Pb}$ & 0.0469 \\
${ }^{207} \mathrm{~Pb} /{ }^{206} \mathrm{~Pb}$ & -0.0154 \\
${ }^{208} \mathrm{~Pb} /{ }^{206} \mathrm{~Pb}$ & 0.0188 \\
& \\
Boxcar averagers & \\
${ }^{208} \mathrm{~Pb} /{ }^{206} \mathrm{~Pb}$ & 0.010 \\
${ }^{207} \mathrm{~Pb} /{ }^{206} \mathrm{~Pb}$ & -0.048 \\
\hline
\end{tabular}

\footnotetext{
${ }^{\text {a }}$ Determined from values for 10,000 counts accumulated isee Table 2). 3)

Determined from average values of calculated ratios /see Table
}

\section{Conclusions}

The excellent resolution of the ICP-TOFMS instrument allows gated detection of individual ion peaks in the time-of-flight mass spectrum. Abundance sensitivity is at least $10^{6}$ for less abundant ions that precede abundant ones, but those that follow abundant peaks would be difficult to detect because of ringing and saturation effects in the MCP detector. We have described three electronic detection methods to perform isotope-ratio analyses via the ICP-TOFMS. Use of commercial boxcar averagers, an in-house-built CFD-gated integration system, and gated ion counting offer precision for these analyses of RSD values between 0.5 and $4 \%$. Gated ion counting showed the best precision and accuracy, but required longer analysis times. In addition, the precision of the gated ion counter approaches that based on counting statistics, which could be $0.1 \%$ or below, provided long enough analysis times are used. This anticipated precision requires more stable gating electronics than are currently utilized in the system. Unfortunately, the counting system has the most limited dynamic range. Commercial boxcar averagers offer acceptable precision; however, the technique (channel switching) used initially to calculate accurate isotope ratios compromises their precision. The CFD-gated integrator system shows the greatest promise for broad dynamic range and excellent precision, although the stability of the present integrators and gates must be improved. Mass discrimination in the ICP-TOFMS is severe compared to what has been reported for other simultaneous mass spectrometers [8], presumably because of detection-system bias.

In summary, the ICP-TOFMS offers the potential for isotope-ratio precision comparable to or better than that reported for quadrupole ICP-MS instruments. However, limitations in the current detection systems preclude very precise measurements. Because the TOFMS extracts all ions simultaneously from the ICP, one would expect ratioing techniques to exhibit even better precision than those obtained with sequential mass spectrometers. Moreover, this improved precision should be available for all ratios across the atomic mass range at once. Because the gated ion-counting method is at the limit of counting statistics, one would expect that greater stability in the timing gates and integrators used in analog methods would afford precision levels better than those seen with scanning instruments. As a consequence, more precise methods of analog detection will be investigated in the future. Finally, the ultimate isotope-ratio analysis method for ICP-TOFMS would be one in which analog signals for all selected ions could be analyzed simultaneously in a multichannel system. Such a setup would take best advantage of the speed and signal information generated by the TOFMS.

\section{Acknowledgments}

This research was funded in part by the National Institutes of Health through grant R01 GM48653.

\section{References}

1. Krause, P.; Kriews, M.; Kannecker, W.; Grabe-Schonberg, C.-D.; Kersten, M. Fresenius J. Anal. Chem. 1993, 347, 324-329.

2. Gregoire, D. C. Prog. Analyt. Spectrosc. 1989, 12, 433-452.

3. Begley, I. S.; Sharp, B. L. I. Anal. Atom. Spectrom. 1994, 9, $171-176$.

4. Furuta, N. J. Anal. Atom. Spectrom. 1991, 6, 199-203.

5. Ketterer, M. E. 1. Anal. Atom. Spectrom. 1992, 7, 1125-1128.

6. Russ, G. P.; Bazan, J. M. Spectrochim. Acta 1987, 42B, 49-62.

7. Longreich, H. P.; Fryer, B. J.; Strong, D. F. Spectrochim. Acta 1987, 42B, 39-48.

8. Walder, A. J.; Freedman, P. A. I Anal. Atom. Spectrom. 1992, 7, $571-575$.

9. Walder, A. J.; Platzner, I.; Freedman, P. A. I. Anal. Atom Spectrom. 1993, 8, 19-23.

10. Walder, A. J.; Koller, D.; Reed, N. M.; Hutton, R. C.; Freedman, P. A. I. Anal. Atom. Spectrom. 1993, 8, 1037-1041.

11. Green, L. W.; Macdonald, R. G.; Sopchyshyn, F. C. Anal. Instrum. 1988, 17, 195-214.

12. Fassett, J. D.; Walker, R. J.; Travis, J. C.; Ruegg, F. C. Int. J. Mass Spectrom. Ion Processes 1987, 75, 111-126.

13. Smith, D. H.; Donohue, D. L.; Young, I. P. Int. I. Mass Spectrom. Lon Processes 1985, 65, 287-297.

14. Simons, D. S. Int. I. Mass Spectrom. Ion Processes 1984, 55, $15-30$.

15. Short, R. T.; Todd. P. J. Isotope ratios using time-of-flight secondary ion mass spectrometry. Presented at the $42 \mathrm{nd}$ ASMS Conference on Mass Spectrometry and Allied Topics; Chicago. IL, May 29-June 3, 1994; talk TOD 10:30.

16. Myers, D. P.; Li, G.; Yang, P.; Hieftje, G. M. J. Am. Soc. Mass Spectrom. 1994, 5, 1008-1016.

17. Myers, D. P.; Li, G.; Mahoney, P. P.; Hieftje, G. M. J. Am. Soc. Mass Spectrom. 1995, 6, 400-410.

18. Myers. D. P.; Li, G.; Mahoney, P. P.; Hieftje, G. M. I. Am. Soc. Mass Spectrom. 1995, 6, 411-420.

14. Yang. ['; Myers, D. P.; Li, G.; Hieftje, G. M. Appl. Spectrosc. $1995,49,660-664$ 